\title{
Biochemical characterization of some cyanobacterial strains from salt marshes of the Venice Lagoon
}

\author{
A. A. Sfriso • D. Marchetto • M. Gallo • F. Baldi
}

Received: 28 January 2013 /Revised and accepted: 17 July 2013 /Published online: 5 September 2013

(C) Springer Science+Business Media Dordrecht 2013

\begin{abstract}
Three different strains of filamentous cyanobacteria, Tychonema, Limnothrix, and Pseudoanabaena, were selected among the fastest growing taxa collected in the salt marshes of Venice Lagoon and were grown in laboratory for growth rate determination and biochemical characterization of chlorophyll$a$, total proteins, total carbohydrates, and exopolysaccharides. Experiments were carried out both in liquid medium and two different substrates: artificial plant protection fabric and ground indigenous shells. Cyanobacterial behavior was recorded to better understand colonization of natural and new artificial marshes.
\end{abstract}

Keywords Cyanobacteria · Salt marshes · Growth rates · Polysaccharides · Venice Lagoon

\section{Introduction}

In recent years, several studies involving cyanobacteria have focused on practical applications to solve environmental problems such as soil and sediment reclamation, bioremediation, and stabilization (Stal 2010; Prochnow et al. 2002; Bender and Phillips 2004).

Cyanobacteria are pioneer species with good adaptive skills, able to colonize barren sediments and soils, fix nitrogen and carbon, and regulate moisture-producing exopolymeric secretions: bacterial capsules, sheaths, and slime of hydrophilic polysaccharides (Decho and Lopez 1993) with variable water content that change with environmental conditions and strain type. These polysaccharides act as protection from sudden osmotic changes and desiccation (Klock et al. 2007), allowing cell adhesion to substrata. In highly variable temperature, salinity, and humidity environments such as salt-marshes,

\footnotetext{
A. A. Sfriso $(\bowtie) \cdot D$. Marchetto $\cdot$ M. Gallo $\cdot$ F. Baldi Dipartimento di Scienze Molecolari e Nanosistemi, Cà Foscari University of Venice, Dorsoduro 2137, 30123 Venice, Italy e-mail: asfriso@hotmail.it
}

cyanobacterial mats, often entangled with colonial diatoms and benthic macroalgae, cover the surface of rising marshes preventing erosion and providing shelter and nourishment for seeds and sprouts starting plant succession (Booth 1941).

In the Venice Lagoon, actions are in place to recreate saltmarshes that have disappeared due to erosion processes, amounting to more than $50 \%$ from 1927 to 2002 (Sarretta et al. 2009). The sediments, mixed with lagoon water, are placed in enclosures of wooden poles or bags filled with clam shells and are allowed to settle. This preliminary study has the aim of selecting indigenous cyanobacterial strains that colonize the natural salt-marshes, covering surface sediment with dense filament mats and exopolysaccharides, in order to select the most suitable taxa useful for stabilization. For this purpose, the growth rates of the most common filamentous taxa and their composition in terms of total carbohydrate (CHO), exopolysaccharides (EPS), and total protein (PRT) content have been studied in liquid and solid substrate media. The objective was to select the fastest growing taxa with the highest EPS production, important features for sediment stabilization (Underwood 1997).

\section{Materials and methods}

Sampling, isolation and characterization

Cyanobacterial mats were sampled in two different marshes of the North Venice Lagoon (Fig. 1), in front of the estuary of the Dese River: the first sample from east of Burano Island $\left(45^{\circ} 29^{\prime} 08.3^{\prime \prime} \mathrm{N}, 12^{\circ} 26^{\prime} 08.7^{\prime \prime} \mathrm{E}\right)$ and the second one in the east marsh of Santa Cristina Island $\left(45^{\circ} 30^{\prime} 03.2^{\prime \prime} \mathrm{N}, 12^{\circ} 28^{\prime} 06.2^{\prime \prime} \mathrm{E}\right)$. Samples were cut with a sterile knife and saved in clean plastic bags (Wirl-Pak) for transport to the laboratory.

All the glassware was autoclaved and all sample transfers were done under a laminar flow hood to reduce biological contamination. Cyanobacteria were isolated by transferring a few patches of cyanobacterial mat with a scalpel onto ASNIII 
Fig. 1 North Venice Lagoon with sampling sites

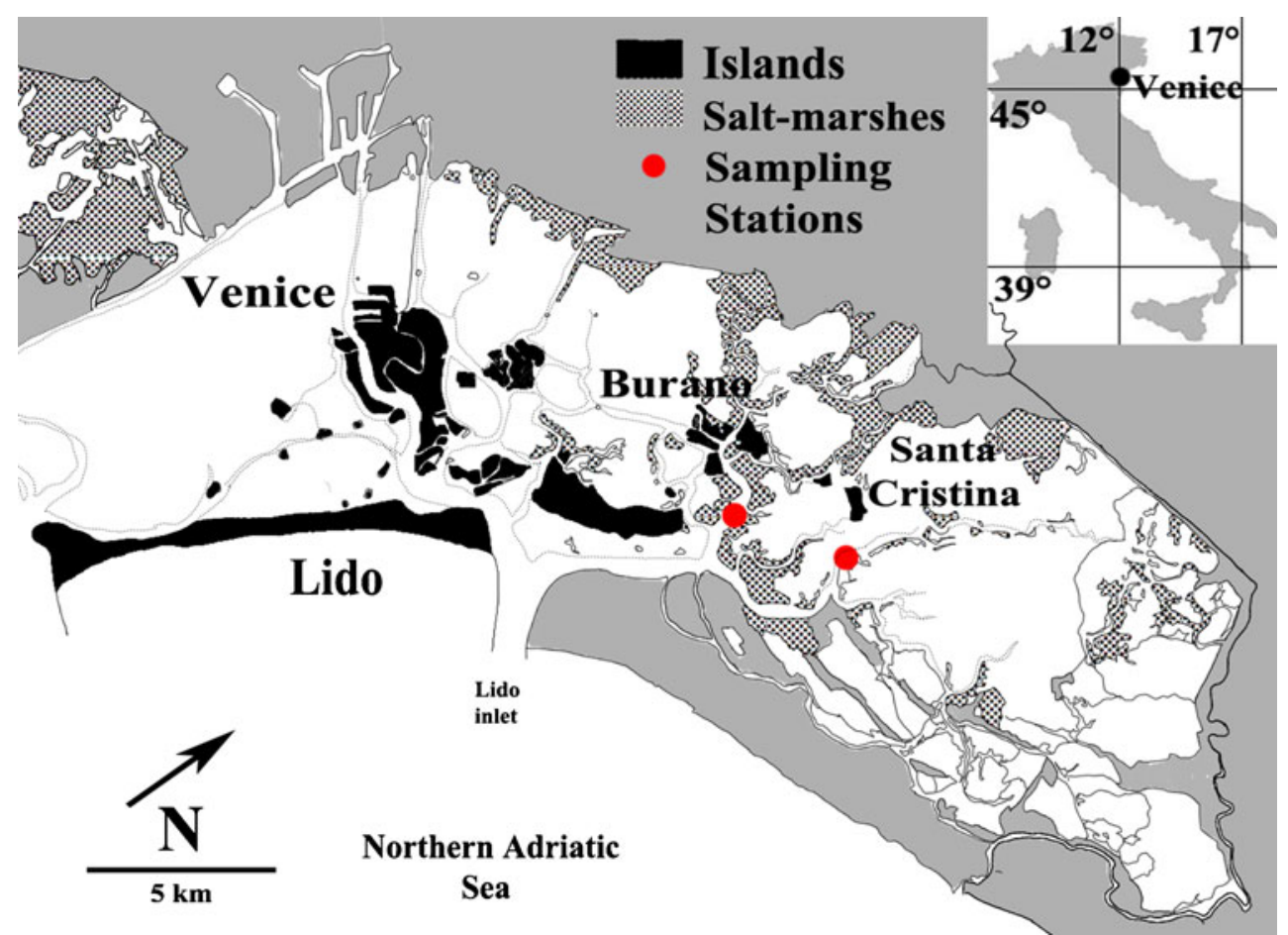

sterilized medium (Waterbury and Stainer 1981) with $1.2 \%$ agar. Incubation was at an average room temperature of $22^{\circ} \mathrm{C}$, under continuous light of $20 \mu \mathrm{mol}$ photons $\mathrm{m}^{-2} \mathrm{~s}^{-1}$ until small sections of the newly grown filaments were transferred onto Petri plates with fresh medium (Stainer et al. 1971; Ferris and Hirsch 1991). The same operation was repeated a number of times, checking the achieved purity in each passage until sections of strains with good cleanness were transferred to liquid ASNIII medium for biochemical characterization experiments. These stock cultures, maintained in the same conditions previously used for isolation, were identified according to Anagnostidis and Komarek's (1985) approach to cyanophytes, Komarek's illustrated tables (http://www. cyanodb.cz), and Rippka's (1988) classification.

\section{Cyanobacterial growth}

The three fastest growing cyanobacterial strains were grown on three different substrata: liquid ASNIII medium (Waterbury and Stainer 1981), plant protection fabric (a polyester cloth), and ground bivalve shells of Acanthocardia tubercolata Linnaeus.

\section{Liquid medium}

For the growth in liquid medium, the isolated strains were dispersed with light shaking for one night and diluted to the desired amount. This stock solution was poured in 21 test tubes with caps for sterile culture, $9 \mathrm{~mL}$ in each. Three tubes of each strain were incubated for 7 days on a rotating drum, with a light exposition of $70 \mu \mathrm{mol}$ photons $\mathrm{m}^{-2} \mathrm{~s}^{-1}$, in light/dark cycles of
$12 \mathrm{~h}$, at an average room temperature of $22^{\circ} \mathrm{C}$. During growth, an amount from each test tube was used for total chlorophyll- $a$ (Chl-a), EPS, PRT, and CHO quantification, to have three biological replicates for analyses, with seven points to observe trend curves and growth rates of biochemical parameters. Chl- $a$ and EPS extraction was performed the same day, while the three samples for either PRT and CHO were kept frozen until analysis.

\section{Solid substrate}

The growth experiment on the plant protection fabric and bivalve shells was carried out in replicates in the same environmental conditions of the liquid experiment, in order to quantify the amounts of Chl- $a$, EPS, PRT, and CHO on two different solid substrata after an empirically chosen time based on the growth in the liquid. For each strain, two sterile flasks for cell culture were used, one with a single layer of plant protection fabric on the bottom and the second one with a layer of ground shells, $20 \mathrm{~g}$ in weight with size ranging between 1 and $5 \mathrm{~mm}$. After 14 days of growth in the same environmental conditions, the colonized substrate was vigorously shaken and vortexed to detach and recover cyanobacterial mats for analysis, verifying the complete detachment of the cyanobacterial filaments under a stereomicroscope.

\section{Biochemical measurements}

Chl- $a$ determination, following Lorenzen's (1967) method, was used as an indirect biomass measurement. The culture sample was centrifuged for $10 \mathrm{~min}$ at $3,660 \times \mathrm{g}$ and the pellets 

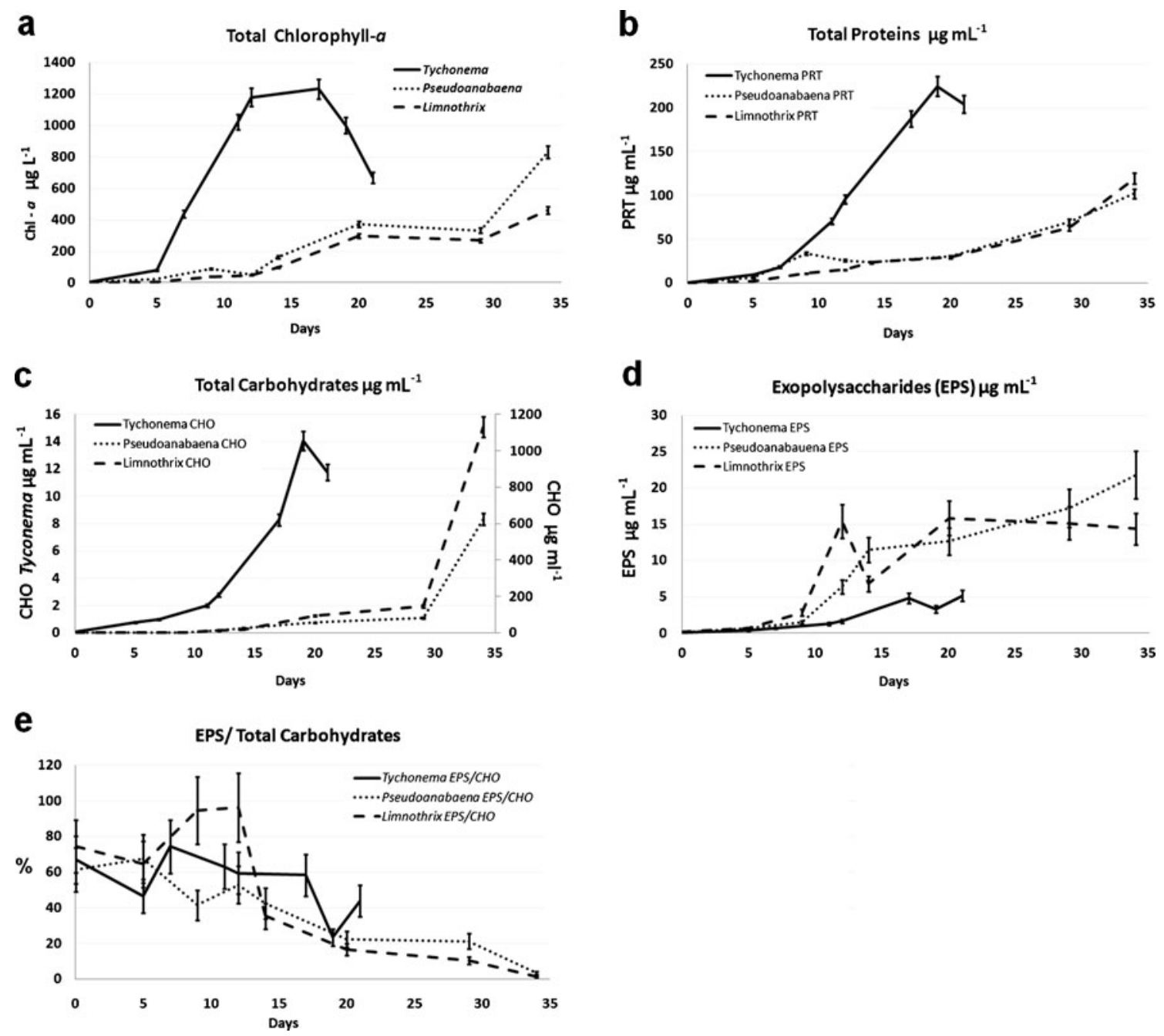

Fig. 2 Cyanobacterial growth (Chl-a), PRT, CHO, and EPS production and EPS/CHO ratios in the experimental design in liquid medium

were extracted with acetone $90 \% \mathrm{v} / \mathrm{v}$ and cold-sonicated for $20 \mathrm{~min}$, completing extraction overnight. The solution was centrifuged again and the supernatant was poured off into a quartz cuvette for spectrophotometric reading at 750 and $665 \mathrm{~nm}$, followed by acidification with few drops of $0.1 \mathrm{~N}$ $\mathrm{HCl}$, and a new reading at $665 \mathrm{~nm}$.

$\mathrm{CHO}$ determination was done using the phenol-sulfuric acid method (Dubois et al. 1956) modified by Rao and Pattabiraman (1989). Glucose solution was used as standard for the calibration curve. The sample was poured into a Pyrex test tube and centrifuged for $10 \mathrm{~min}$ at $3,660 \times \mathrm{g}$, then pellets were digested in $3 \mathrm{~mL} \mathrm{H}_{2} \mathrm{SO}_{4} 72 \%$ overnight. The digested sample was centrifuged again and $1 \mathrm{~mL}$ was transferred into a new Pyrex tube with $5 \mathrm{~mL} \mathrm{H}_{2} \mathrm{SO}_{4} 96 \%$. After $15 \mathrm{~min}$ at $105^{\circ} \mathrm{C}$ and few minutes for cooling, $1 \mathrm{~mL}$ of $5 \% \mathrm{w} / \mathrm{v}$ phenol was added and the absorbance was read at $485 \mathrm{~nm}$.

EPS fractions of exopolymeric substances were extracted and determined as $\mathrm{CHO}$. The culture sample was centrifuged and the pellets were extracted with $100 \mathrm{mM}$ EDTA for 15 min under constant agitation at $20^{\circ} \mathrm{C}$, using the extraction method suggested by Underwood et al. (1995), and recovered by precipitation adding cold ethanol (Klock et al. 2007).

Estimation of PRT was obtained according to Bradford's (1976) micro-method recommended by Bio-Rad, the reagent supplier. Bovine serum albumin was used as standard for the calibration curve. The sample was centrifuged and pellets were extracted with an alkaline solution of $0.5 \mathrm{~N} \mathrm{NaOH}$ and sonication for $2 \mathrm{~h}$ at $40{ }^{\circ} \mathrm{C}$ (Nunn and Keil 2006), completed overnight. The sample was centrifuged again and $0.1 \mathrm{~mL}$ was mixed with $0.7 \mathrm{~mL}$ milli-Q water and $0.2 \mathrm{~mL}$ "Concentrate Bio-Rad Protein Assay Dye reagent" Coomassie Brilliant Blue G solution. Polycarbonate cuvette was used for spectrophotometric reading at $595 \mathrm{~nm}$.

Statistical analyses

All results obtained by the growth and chemical composition of the three cyanobacterial strains were analyzed by determining Spearman's coefficients using Excel 2000. 


\section{Results}

The three most rapidly growing strains, isolated from cyanobacterial mats collected in Venice salt-marshes, were classified as Tychonema Anagnostidis and Komarek (Phormidiaceae), Pseudoanabaena Lauterborn (Pseudoanabaenaceae), and Limnothrix Meffert (Pseudoanabaenaceae). All the three genera are filamentous.

Tychonema In agar growth was slow, giving a slightly visible green halo in the furthest millimeters around the inoculated patch. In ASNIII liquid medium growth quickened; a few days after inoculation voluminous green flakes were already clearly visible and took up the whole liquid volume in 12 days. In 2 weeks of growth, the strain was able to colonize the whole of the substrata, the plant protection fabric and ground shells, with a tough biofilm. Microscopic investigation revealed isodiametric cells, 3.4-5.1 $\mu \mathrm{m}$ in width, separated by slight constrictions, lined up in straight trichomes, solitary and gathered in mats. Granules and necridia were present in the cells. Filaments exhibited slight motility and there was an absence of heterocysts, branches, sheaths, caliptra, and gas vesicles.

Limnothrix In agar, filament growth was rapid and the Petri dish was half-colonized in 10 days. In liquid medium, growth was slower with slightly visible green plates on the liquid surface after 2 weeks. In the same period, the strain was able to colonize the whole of the substrata with a tough biofilm. Microscopy revealed cylindrical, elongated cells, $2-3 \mu \mathrm{m}$ in width, lined up in slightly curved trichomes gathered in mats. Mucilaginous envelopes, granules, and gas vesicles were present, whereas heterocysts, constrictions, branches, and caliptra were absent.

Pseudoanabaena In agar, growth was rapid and the whole Petri dish was colonized in 10 days. The strain was not further investigated in the substrate growth experiment because of the biochemical and growth rate similarities in liquid medium to Limnothrix and the slower EPS and $\mathrm{CHO}$ production. The strain had cylindrical, stretched cells, $2-3 \mu \mathrm{m}$ in width, separated by clear constrictions and lined up in trichomes braided in pairs and gathered in thick mats. Peripheral thylakoids were present whereas heterocysts, branches, sheats, caliptra and gas vesicles were lacking.

\section{Liquid growth kinetics}

Tychonema displayed an exponential growth in the first 12 days (reaching Chl- $a$ : 1,234 $\mathrm{g} \mathrm{L}^{-1}$ in 17 days) in liquid medium (Fig. 2a) while Limnothrix and Pseudoanabaena reached a quarter of the Tychonema biomass in almost the same time (Chl- $a$ : 372 and $299 \mu \mathrm{g} \mathrm{L}^{-1}$, respectively, after
20 days) displaying significant differences in $\mathrm{CHO}$, PRT, and EPS production (Fig. 2b-d).

The biochemical analysis of the single strains revealed significant relationships between $\mathrm{CHO}$ and PRT in both Tychonema and Limnothrix and Pseudoanabaena, with a CHO logaritmic increase (Table 1).

EPS were higher in the first days of growth of all the strains (from 60 to $80 \%$ of $\mathrm{CHO}$ ), reaching a stationary phase in 2 weeks (Fig. 2e), Tychonema EPS production was instead, after the same time, one-third the amount recorded in Limnothrix and Pseudoanabaena (Fig. 2d). After 1 month, Pseudoanabaena and Limnothrix suddenly displayed an increase in Chl- $a$ and PRT and high CHO production, leaving EPS unchanged.

Growth on substrata

The substrate experiment displayed an increase of Chl- $a$ in the shells compared to the artificial fabric. In addition, there were extremely lower values of biomass increment in comparison to liquid cyanobacterial growth in all the strains (Fig. 3). PRT had almost the same increase in Tychonema on both the solid substrata, while Limnothrix increased PRT production on the shells by three times compared to the artificial fabric. In contrast, $\mathrm{CHO}$ increased mainly on the plant protection fabric, twice that on the shells, while EPS

Table 1 Chl- $a$, CHO, PRT, and EPS production correlation analysis between the single genera

\begin{tabular}{|c|c|c|c|c|}
\hline & Chl- $a$ tot & $\mathrm{CHO}$ & PRT & EPS \\
\hline \multicolumn{5}{|c|}{ Tychonema } \\
\hline \multicolumn{5}{|c|}{ Chl- $a$ tot } \\
\hline $\mathrm{CHO}$ & 0.60 & & & \\
\hline PRT & 0.76 & 0.96 & & \\
\hline EPS & 0.69 & 0.87 & 0.93 & \\
\hline \multicolumn{5}{|c|}{$p<0.05$ per $r>|0.76|$} \\
\hline \multicolumn{5}{|c|}{ Limnothrix } \\
\hline \multicolumn{5}{|c|}{ Chl- $a$ tot } \\
\hline $\mathrm{CHO}$ & 0.82 & & & \\
\hline PRT & 0.92 & 0.92 & & \\
\hline EPS & 0.73 & 0.41 & 0.61 & \\
\hline \multicolumn{5}{|c|}{$p<0.05$ per $r>|0.73|$} \\
\hline \multicolumn{5}{|c|}{ Pseudoanabaena } \\
\hline \multicolumn{5}{|c|}{ Chl- $a$ tot } \\
\hline $\mathrm{CHO}$ & 0.92 & & & \\
\hline PRT & 0.91 & 0.85 & & \\
\hline EPS & 0.89 & 0.73 & 0.87 & \\
\hline \multicolumn{5}{|c|}{$p<0.05$ per $r>|0.73|$} \\
\hline
\end{tabular}

Significant values in bold

Chl-a Chlorophyll-a, CHO carbohydrates, PRT proteins, EPS exopolyschaccarides 
Fig. 3 Concentration ratios of Chl- $a$, PRT, CHO, EPS production after 14 days on fabric, shell, and liquid media a
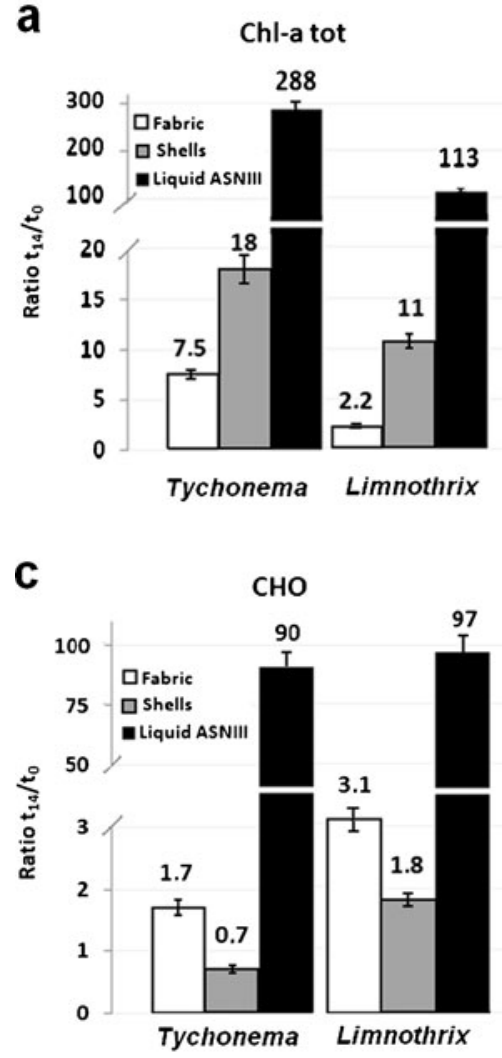
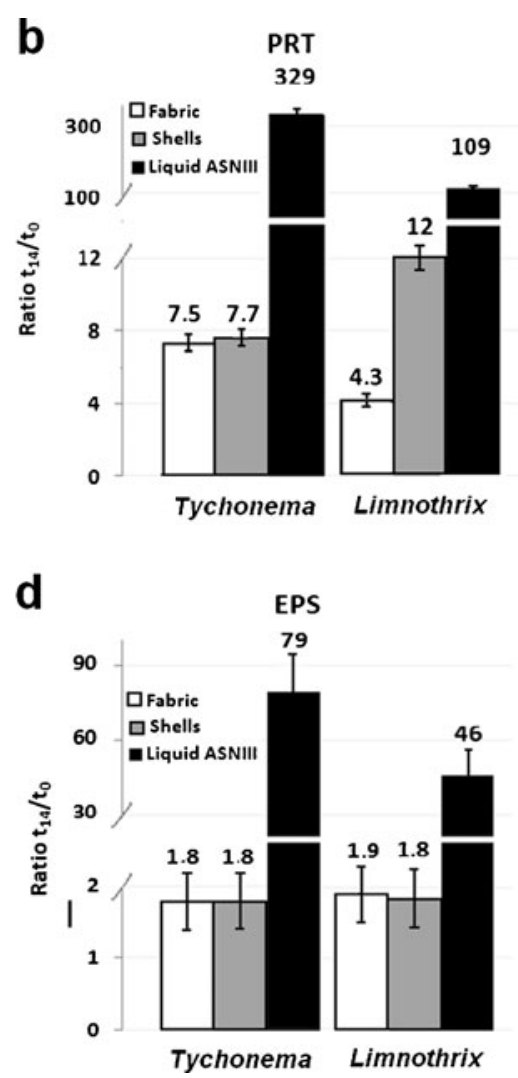

production was almost the same on both substrata for both strains. Both the experimental flasks retained the substrate on the bottom after being turned upside down.

\section{Discussion}

The three fastest growing cyanobacterial strains were selected from the intertidal surface sediment of the Venice Lagoon to study growth and biochemical development on new, still uncolonized, substrata and in liquid medium to better understand the different growth behavior of these cyanobacteria: as free-floating trichomes and as benthic mats bound to substrates. The selected strains displayed opposite behavior. Tychonema (Phormidiaceae) showed a good dispersion in liquid medium, growing voluminous green flakes high in PRT and Chl- $a$ but low in CHO production. Limnothrix and Pseudoanabaena (Pseudoanabaenaceae) were strongly attached to all the glassware and grew slowly though with biofilms and green plates on the liquid surface, with high EPS/CHO ratios in the exponential growth phase. The sudden increase of $\mathrm{CHO}$ after 1 month of growth, missing in the EPS production, suggests an increase in storage polysaccharides, also clearly visible as granules in the microscope, a cellular response probably related to a nutrient depletion (Schwarz and Forchhammer 2005).
The liquid growth was shown to be always a lot more productive in biomass compared to growth on substrata, a condition probably due to a three dimensional provision of light, nutrients, and carbon dioxide. The slight motion emulating the natural conditions of water waves stimulated growth of free trichomes and their dispersion, also explaining the better growth on liquid medium. The shells were shown to be the better growing support as revealed by the PRT and Chl- $a$ growth ratio at 14 days (Fig. 2a, b), also because of the higher surface area of the ground shell. The polyester cloth slightly enhanced CHO production (Fig. 2c), with an increase in EPS that was almost the same for both the substrata and strains (Fig. 2d), suggesting a greater production of cellular carbohydrates on the plant protection fabric.

\section{Conclusions}

Both the selected strains of Tychonema and Limnothrix produced high ratios of EPS/CHO (maximum values reached in the first 14 days: $74 \%$ for Tychonema and $96 \%$ for Limnothrix) and thick mats. These strains, in stable laboratory conditions, display useful features for an artificial colonization of new saltmarsh border confinements. These colonized supports can became spreading hotspots favoring organic carbon and nitrogen 
enrichment of adjacent soils and the growth of a living texture that retains sediments, as revealed by the strong attachment of colonized substrata to the experimental flasks, both having the same EPS production (an increment of about two times in both the substrata and strains). The rapid growth of Tychonema and spreading in the liquid medium, as shown by the increment in PRT (329 times in 14 days) and Chl- $a$ (288 times in 14 days), can help to cover new incoherent grounds favoring a later stabilization of substrata, while the slower colonization of Limnothrix with thick mats can facilitate sediment stabilization and enrichment with polysaccharides useful for other microorganism growth and macrophyte attachment.

\section{References}

Anagnostidis K, Komarek J (1985) Modern approach to the classification system of cyanophytes. Arch Hydrobiol 71:291-302

Bender J, Phillips P (2004) Microbial mats for multiple applications in aquaculture and bioremediation. Bioresour Technol 94:229-238

Booth WE (1941) Algae as pioneers in plant succession and their importance in erosion. Ecology 221:38-46

Bradford MM (1976) A rapid sensitive method for the quantitation of microgram quantities of protein utilizing the principle of protein-dye binding. Anal Biochem 81:481-484

Decho WA, Lopez GR (1993) Exopolymer microenvironments of microbial flora: multiple and interactive effects on trophic relationships. Limnol Oceanogr 388:1633-1645

Dubois M, Gilles KA, Hamilton JK, Rebers PA, Smith F (1956) Colorimetric method for determination of sugars and related substances. Anal Biochem 28:350-356

Ferris MJ, Hirsch CF (1991) Method for isolation and purification of cyanobacteria. Appl Environ Microbiol 575:1448-1452
Klock JH, Wieland A, Seifert R, Michaelis W (2007) Extracellular polymeric substances (EPS) from cyanobacterial mats: characterization and isolation method optimization. Mar Biol 152:1077-1085

Lorenzen CJ (1967) Determination of chlorophyll and pheopigments: spectrophotometric equations. Limnol Oceanogr 12:343-346

Nunn BL, Keil RG (2006) A comparison of non-hydrolytic methods for extracting amino acids and proteins from coastal marine sediments. Mar Chem 98:31-42

Prochnow J, Schweim C, Koengeter J (2002) Simulation of biogenic sediment stabilization by heterotrophic bacteria in annular flume. Proc Mar Sci 5:393-407

Rao P, Pattabiraman TN (1989) Reevaluation of the phenol-sulfuric acid reaction for the estimation of hexoses and pentoses. Anal Biochem 1811:18-22

Rippka R (1988) Isolation and purification of cyanobacteria. Methods Enzymol 167:3-28

Sarretta A, Pillon S, Molinaroli E, Guerzoni S, Fontolan G (2009) Sediment budget in the Lagoon of Venice, Italy. Cont Shelf Res 30:934-949

Schwarz R, Forchhammer K (2005) Acclimation of unicellular cyanobacteria to macronutrient deficiency: emergence of a complex network of cellular responses. Microbiology 151:2503-2514

Stainer RY, Kunisawa R, Mandel M, Cohen-Bazire G (1971) Purification and properties of unicellular blue-green algae (order Chroococcales). Bacteriol Rev 32:171-205

Stal LJ (2010) Microphytobenthos as a biogeomorphological force in intertidal sediment stabilization. Ecol Eng 362:236-245

Underwood GJC, Paterson DM, Parkes RJ (1995) The measurements of microbial carbohydrates exopolymers from intertidal sediments. Limnol Oceanogr 40:1243-1253

Underwood GJC (1997) Microalgal colonization in a saltmarsh restoration scheme. Estuar Coast Shelf Sci 44:471-481

Waterbury JB, Stainer RY (1981) Isolation and growth of cyanobacteria from 214 marine and hypersaline environments. In: Starr HP, Stulp H, Truper HG, Balows A, Schleeper HG (eds) The prokaryotes: a handbook on habitats, isolation, and identification of bacteria, vol 1 . Springer, Berlin, pp 221-223 